\title{
Ärztegesellschaft Baselland
}

Walter Frommenwiler

Ausschussmitglied

Ärztegesellschaft BL

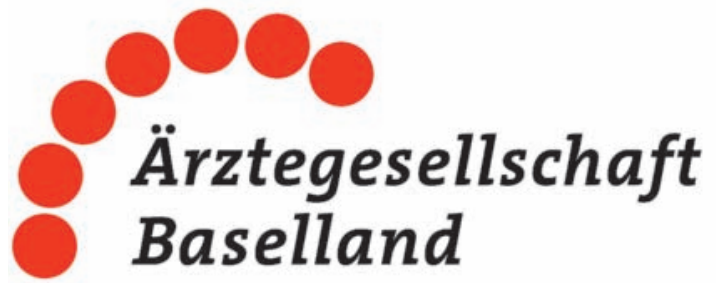

Wohl kurz nach der Kantonstrennung von 1833 entstand eine erste Baselbieter Ärztevereinigung. Seit den Anfängen mit einer Handvoll Mitgliedern hat sich unsere Gesellschaft zu einer modernen Standesvertretung und Dienstleistungsorganisation für die ganze Ärzteschaft des Halbkantons Basel-Landschaft entwickelt.

Das Baselbiet ist heute politisch, wirtschaftlich und kulturell eng mit dem Kanton BaselStadt verflochten. So besteht für die Universität Basel seit kurzem eine gemeinsame Trägerschaft. Im Gesundheitswesen bestehen gemeinsame Spitalplanungen und eine enge Zusammenarbeit zwischen Kliniken in der Stadt und auf der Landschaft. Auch die Ärztegesellschaft Baselland arbeitet eng mit der Medizinischen Gesellschaft Basel zusammen. Sichtbares Zeichen dieser Zusammenarbeit ist die gemeinsame Herausgabe unseres Kommunikationsorgans, der «Synapse».

Eine Hauptaufgabe unserer Gesellschaft ist die Organisation des ambulanten Notfalldienstes. Aufgrund der veränderten Bedürfnisse der Bevölkerung und der veränderten Ärztedemographie werden neue Konzepte für diese Dienstleistung erarbeitet. Die Gesellschaft betreibt ferner eine Ombudsstelle, an die sich Patientinnen und Ärzte bei gegenseitigen Problemen wenden können.
Regelmässige Kontakte mit dem zuständigen Regierungsrat, dem Kantonsarzt und den Parlamentariern ermöglichen immer wieder pragmatische, für beide Seiten sinnvolle Lösungen, beispielsweise bei der Anwendung des Zulassungsstopps. Die etablierte Zusammenarbeit mit der Politik hat 2007 eine für uns erfolgreiche Revision des Gesundheitsgesetzes mit Weiterführung der Selbstdispensation ermöglicht. Der Regierungsrat hat erfreulicherweise beschlossen, die Praxisassistenz bei Hausärzten zu fördern.

Momentan läuft ein Taxpunktwert-Festsetzungsverfahren beim Regierungsrat des Kantons Basel-Landschaft, nachdem für 2008 mit santésuisse kein Taxpunktwert vereinbart werden konnte. Wegen unterschiedlicher Auffassungen über die Vertragsauslegung hat die Ärztegesellschaft Baselland mittlerweile die LeiKoV-Vertragsanhänge gekündigt. Tariffragen können dank der Erfahrung unseres Tarifdelegierten Dr. med. Roland Schwarz und des hohen Anteils unserer Mitglieder, die ihre Fakturierungsdaten an ein Trustcenter liefern, aufgrund solider Daten entschieden werden. Damit bestehen unsererseits die Voraussetzungen, in Zukunft zu vernünftigen Tarifvereinbarungen Hand bieten zu können. Mit Syndata, dem ärzteeigenen Trustcenter, können wir unsere Mitglieder beim Controlling ihrer Praxisführung und bei Wirtschaftlichkeitsverfahren beraten.

Durch ihre Kampagne zum Erhalt der Selbstdispensation hat die Ärztegesellschaft Baselland gezeigt, dass sie sich politisch Gehör verschaffen kann. Wir kämpfen dafür, dass wir weiterhin unseren Patientinnen und Patienten unsere Dienste in gewohnter Qualität anbieten können.
Dr. med. Walter Frommenwile

Bahnhofstrasse 20

CH-4402 Frenkendorf 